\title{
Kummer Surfaces: 200 Years of Study
}
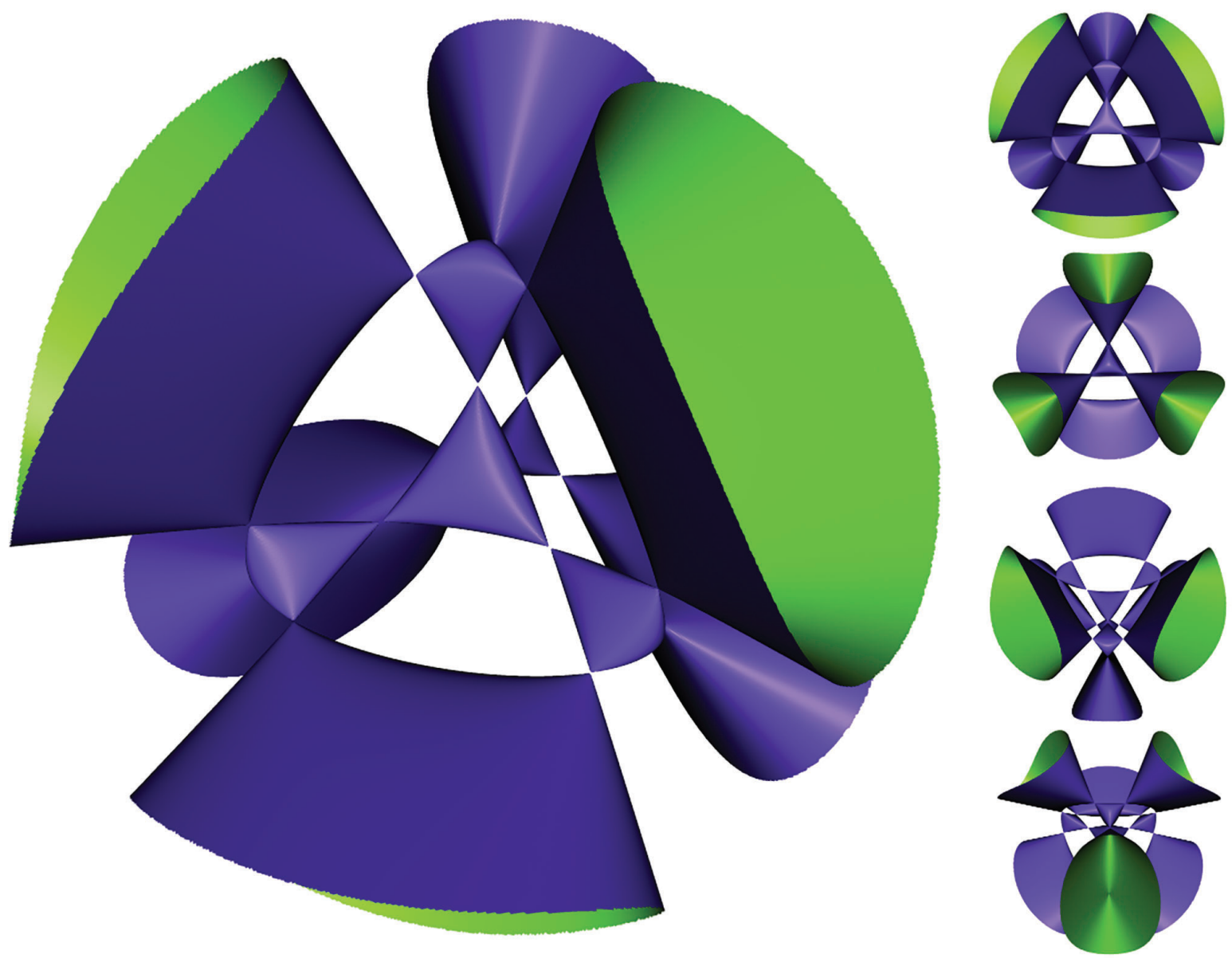

Igor Dolgachev

The fascinating story about the Kummer surface starts from the discovery by Augustin-Jean Fresnel in 1822 of the equation describing the propagation of light in an optically biaxial crystal [Fre]. Biaxial crystals are an example of

Igor Dolgachev is a professor of mathematics, emeritus, at the University of Michigan. His email address is ido1ga@umich.edu.

The article is based on the author's Oliver Club talk at Cornell University delivered on October 10, 2019, exactly 121 years since the first meeting of the club, at which then the faculty member John Hutchinson spoke. As we will see, Hutchinson contributed significantly to the theory of Kummer surfaces.

Communicated by Notices Associate Editor Angela Gibney.

For permission to reprint this article, please contact:

reprint-permission@ams .org.

DOI: https://doi.org/10.1090/noti2168 material where double refraction occurs: a ray of light splits into two, traveling at the same speed along different paths. The speed of light may depend on the coordinates $x=\left(x_{1}, x_{2}, x_{3}\right)$ of a point and the unit direction vector $\xi=\left(\xi_{1}, \xi_{2}, \xi_{3}\right)$. The propagation of light is described by the speed $v(x, \xi)$ at $x$ in the direction $\xi$. We say that the matter is homogeneous if $v$ does not depend on $x$ and we say that it is isotropic if it does not depend on $\xi$. For example, while a student, James Maxwell described a lens that reminded him of the eyes of a fish. Through his fish eye, he found that light is inhomogeneous but isotropic, bending in arcs whose shape depends on where they start and converging to a single point. 


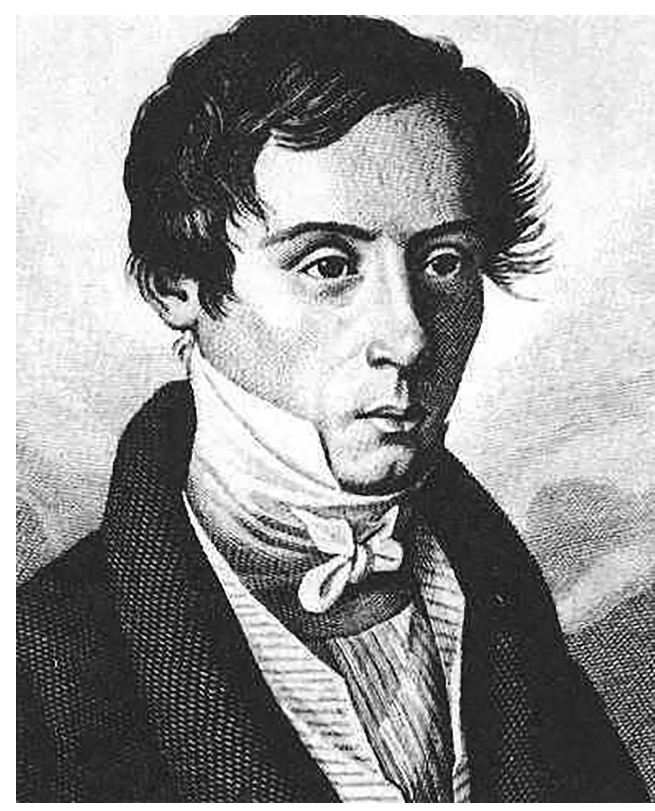

Figure 1. Augustin-Jean Fresnel.

A biaxial crystal gives an example of homogeneous but anisotropic propagation. Fresnel found the equation of the propagation of light in such a crystal to be of the form:

$$
\frac{\xi_{1}^{2}}{a_{1}^{-2}-v^{-2}}+\frac{\xi_{2}^{2}}{a_{2}^{-2}-v^{-2}}+\frac{\xi_{3}^{2}}{a_{3}^{-2}-v^{-2}}=0
$$

where $a_{1}, a_{2}, a_{3}$ are constants describing the property of the crystal (principal refraction indices). By substituting $\left(z_{1}, z_{2}, z_{3}\right)=v\left(\xi_{1}, \xi_{2}, \xi_{3}\right)$, one can rewrite the previous equation as

$$
\begin{aligned}
\frac{a_{1}^{2} z_{1}^{2}}{z_{1}^{2}+z_{2}^{2}+z_{3}^{2}-a_{1}^{2}}+\frac{a_{2} z_{2}^{2}}{z_{1}^{2}+z_{2}^{2}+z^{2}-a_{2}^{2}} & \\
& +\frac{a_{3} z_{3}^{2}}{z_{1}^{2}+z_{2}^{2}+z_{3}^{2}-a_{3}^{2}}=0 .
\end{aligned}
$$

After clearing the denominators and homogenizing, we find an equation of a quartic surface in $\mathbb{P}^{3}$. In $1833 \mathrm{Sir}$ William Hamilton discovered that the surface has four real singular points

$$
\left( \pm a_{3} \sqrt{\frac{a_{1}^{2}-a_{2}^{2}}{a_{1}^{2}-a_{3}^{2}}}, 0, \pm a_{1} \sqrt{\frac{a_{2}^{2}-a_{3}^{2}}{a_{1}^{2}-a_{3}^{2}}}, 1\right)
$$

and also four real planes $\alpha x+\beta y+\gamma z+w=0$ that cut out the surface along a conic (trope-conics), where

$$
(\alpha, \beta, \gamma, 1)=\left( \pm \frac{a_{3}}{a_{2}^{2}} \sqrt{\frac{a_{1}^{2}-a_{2}^{2}}{a_{1}^{2}-a_{3}^{2}}}, 0, \pm \frac{a_{1}}{a_{2}^{2}} \sqrt{\frac{a_{2}^{2}-a_{3}^{2}}{a_{1}^{2}-a_{3}^{2}}}, 1\right)
$$

[Ham37, p. 134]. In fact, as we shall see later, over $\mathbb{C}$, it has additionally 12 nodes and 12 trope-conics.

In 1849 Arthur Cayley proved that Fresnel's wave surface is a special kind of a tetraedroid quartic surface. The latter is characterized by the following property. There are four

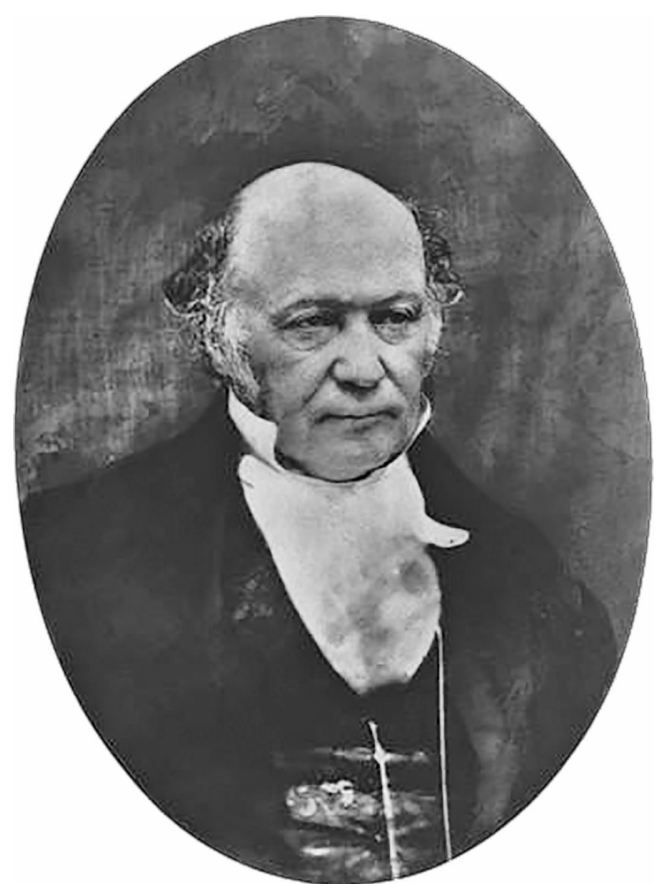

Figure 2. Sir William Hamilton.

planes forming a tetrahedron such that each plane cuts out the surface along a pair of conics. The three vertices of the tetrahedron are conjugate with respect to the two conics lying in the face of the tetrahedron they span. Moreover 16 intersection points of four pairs of conics are singular points of the surface. Cayley also discovered an important property of tetraedroid quartic surfaces: they are projectively self-dual (or reciprocal). Wave surfaces were the subject of study for many famous mathematicians of the 19th century.

Among them were A. Cauchy, J. Darboux, J. MacCullagh, J. Sylvester, and W. Hamilton (see [Lor96, pp. 114115]). A nice modern exposition of the theory of Fresnel's wave surfaces can be found in [Knö86].

Projective equivalence classes of Fresnel's wave surfaces depend on two parameters and as we shall see momentarily, these are examples of Kummer surfaces which are determined by three parameters.

In 1847 Adolph Göpel, using the transcendental theory of theta functions, had found a relation of order four between theta functions of second order in two variables that expresses an equation of a general Kummer surface [Göp47]. To give Göpel's equation, we next briefly discuss theta functions.

Let $T=\mathbb{C}^{g} / \Lambda$ be a compact $g$-dimensional torus, the quotient of $\mathbb{C}^{g}$ by the group of translations $\Lambda$ isomorphic to $\mathbb{Z}^{2 g}$. There are no nonconstant holomorphic functions on $T$ because it is a compact complex manifold; instead one considers nonzero holomorphic sections of a holomorphic line bundle $L$ on $T$. For general $\Lambda$ no line 


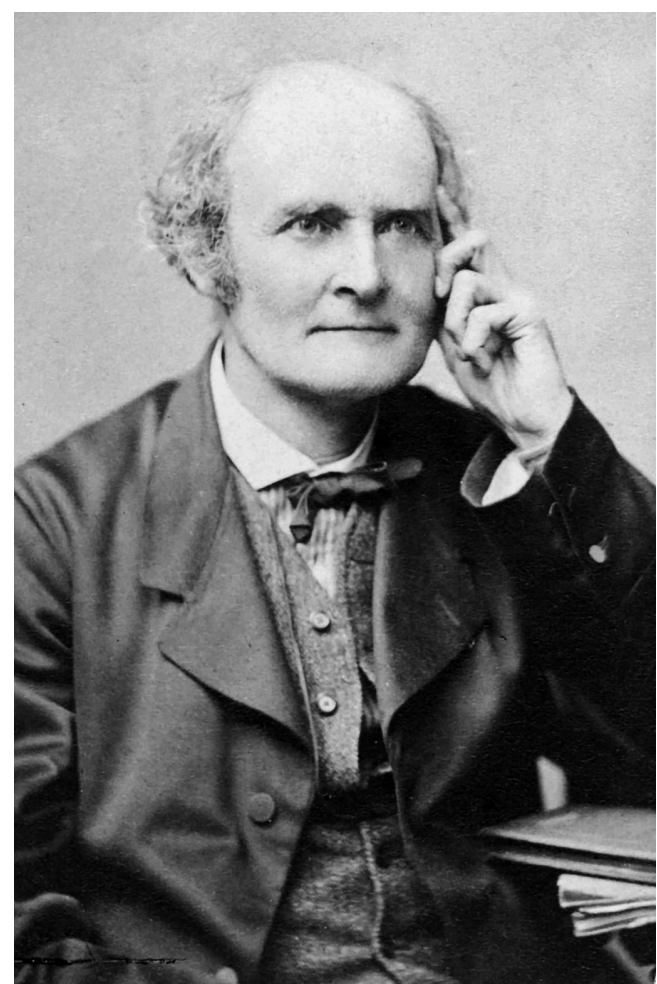

Figure 3. Arthur Cayley.

bundle has enough sections to embed $T$ into a projective space, i.e., $T$ does not admit a structure of a projective algebraic variety. However, in 1857 Bernard Riemann found a condition on $\Lambda$ such that $T$ admits a line bundle $L$ with $\operatorname{dim} \Gamma\left(T, L^{n}\right)=n^{g}$ and whose sections embed $T$ onto a projective space. Such complex tori are now called principally polarized abelian varieties. They depend on $\frac{1}{2} g(g+1)$ complex parameters. Holomorphic sections of $L^{n}$ can be lifted to holomorphic functions on $\mathbb{C}^{g}$ that are $\Lambda$-invariant up to some multiplicative factor. They are called theta functions of order $n$. For $n=1$, such a holomorphic function is the famous Riemann theta function $\Theta(z, \Lambda)$. One can modify Riemann's expression for $\Theta(z, \Lambda)$ to include some parameters which are elements $\left(m, m^{\prime}\right)$ of the group $(\mathbb{Z} / n \mathbb{Z})^{g} \oplus(\mathbb{Z} / n \mathbb{Z})^{g}$, called theta characteristics. They generate the linear space $\Gamma\left(T, L^{n}\right)$.

An example of a principally polarized abelian variety is the Jacobian variety $\operatorname{Jac}(C)$ of a Riemann surface $C$ of genus $g$. Here $\Lambda$ is spanned by vectors $v_{i}=\left(\int_{\gamma_{i}} \omega_{1}, \ldots, \int_{\gamma_{i}} \omega_{g}\right) \in$ $\mathbb{C}^{g}, i=1, \ldots, 2 g$, where $\left(\omega_{1}, \ldots, \omega_{g}\right)$ is a basis of the linear space of holomorphic differential 1 -forms and $\left(\gamma_{1}, \ldots, \gamma_{2 g}\right)$ is a basis of $H_{1}(T, \mathbb{Z})$.

Göpel was able to find a special basis $\left(\theta_{0}, \theta_{1}, \theta_{2}, \theta_{3}\right)$ in the space $\Gamma\left(\operatorname{Jac}(C), L^{2}\right) \cong \mathbb{C}^{4}$ such that the map

$$
\Phi: T \rightarrow \mathbb{P}^{3},\left(z_{1}, z_{2}\right) \mapsto\left(\theta_{0}(z): \theta_{1}(z): \theta_{2}(z): \theta_{3}(z)\right)
$$

satisfies $\Phi\left(-z_{1},-z_{2}\right)=\Phi\left(z_{1}, z_{2}\right)$ and its image $X$ is the set

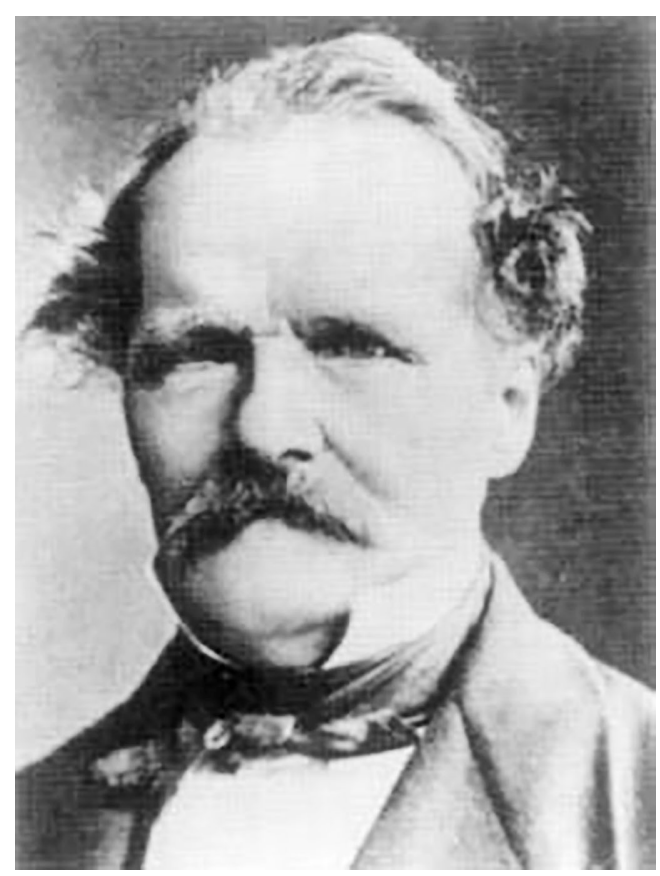

Figure 4. Ernst Kummer.

of zeros in $\mathbb{P}^{3}$ of a quartic polynomial

$$
\begin{aligned}
F= & A\left(x^{4}+y^{4}+z^{4}+w^{4}\right)+2 B\left(x^{2} y^{2}+z^{2} w^{2}\right) \\
& +2 C\left(x^{2} z^{2}+y^{2} w^{2}\right)+2 D\left(x^{2} w^{2}+y^{2} z^{2}\right)+4 E x y z w,
\end{aligned}
$$

where the coefficients $(A, B, C, D, E)$ satisfy a certain explicit equation in terms of theta constants, the values of theta functions at 0 . The images of 16 two-torsion points $\epsilon \in \frac{1}{2} \Lambda / \Lambda \in \mathbb{C}^{2} / \Lambda$ are singular points of $X$. The Abel-Jacobi map $C \rightarrow \operatorname{Jac}(C), x \mapsto\left(\int_{x_{0}}^{x} \omega_{1}, \int_{x_{0}}^{x} \omega_{2}\right) \bmod \Lambda$ embeds $C$ into $\operatorname{Jac}(C)$ and the images of the curves $C+\epsilon=\{c+\epsilon, c \in C\}$ are the 16 trope-conics of $X$.

In 1864 Ernst Kummer had shown that 16-nodal quartic surfaces depend on three complex parameters and Fresnel's wave surface represents only a special case of these quartic surfaces. Kummer proved that such surfaces contain 16 trope-conics which together with 16 nodes form an abstract incidence configuration $\left(16_{6}\right)$ (this means that each node lies on six trope-conics and each trope-conic contains six nodes) [Kum64]. Kummer shows that any 16nodal quartic surface has a tetrahedron with conic-tropes in the faces intersecting at two points on the edges. Moreover, he proved that no vertex can be a node. From this he deduced that there exists a quadric surface that contains the four trope-conics. Using this observation, he found an equation of a general Kummer surface of the form

$$
\begin{gathered}
\left(x^{2}+y^{2}+z^{2}+w^{2}+a(x y+z w)+b(x z+y w)\right. \\
+c(x w+y z))^{2}+K x y z w=0,
\end{gathered}
$$

where $x y z w=0$ is the equation of a chosen tetrahedron 


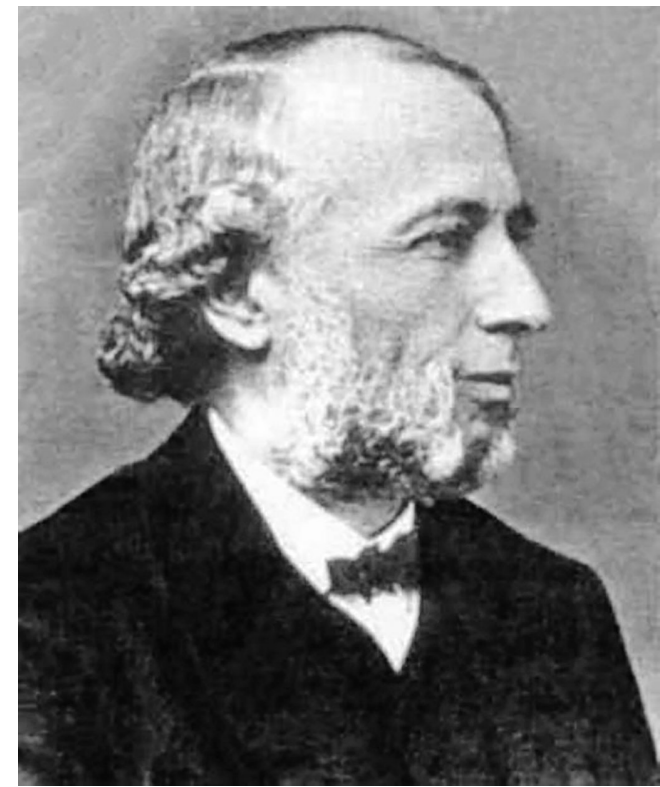

Figure 5. Carl Borchardt.

and $K=a^{2}+b^{2}+c^{2}-2 a b c-1$. Since then any 16-nodal quartic surface became known as a Kummer quartic surface.

It took almost 30 years since Göpel's discovery to realize that the Göpel equation, after a linear change of variables, can be reduced to a Kummer equation. This was done by Carl Borchardt in 1877 [Bor77]. In fact, Göpel's discovery leads to a modern definition of the Kummer surface and its higher-dimensional version, the Kummer variety. One considers any g-dimensional complex torus $T$ and divides it by the involution $z \mapsto-z$; the orbit space is called the Kummer variety of $T$ and is denoted by $\operatorname{Kum}(T)$. It was shown by Andre Weil that $T$ has always a structure of a Kähler manifold. If $T$ admits an embedding into a projective space, then $\operatorname{Kum}(T)$ is a projective algebraic variety with $2^{2 g}$ singular points. Moreover, if $T$ is a principally polarized abelian variety, for example the Jacobian variety of a genus $g$ algebraic curve, one can embed $\operatorname{Kum}(T)$ into the projective space $\mathbb{P}^{2^{g}-1}$ to obtain a self-dual subvariety of degree $2^{g-1} g$ ! with $2^{2 g}$ singular points and $2^{2 g}$ trope hyperplanes. If $g \geq 3$, each intersects it with multiplicity 2 along a subvariety isomorphic to the Kummer variety of a $(g-1)$-dimensional principally polarized abelian variety.

There is also a generalized Kummer variety of complex dimension $2 r$ introduced by Arnaud Beauville in 1983. It is a nonsingular compact holomorphic symplectic manifold birationally isomorphic to the kernel of the addition map $A^{(r+1)} \rightarrow A$, where $A$ is an abelian variety of dimension 2 and $A^{(r+1)}=A^{r} / \mathfrak{S}_{r+1}$ is its symmetric product. The family of such surfaces is one of a few known examples of complete families of compact holomorphic symplectic manifolds of dimension $2 r$.

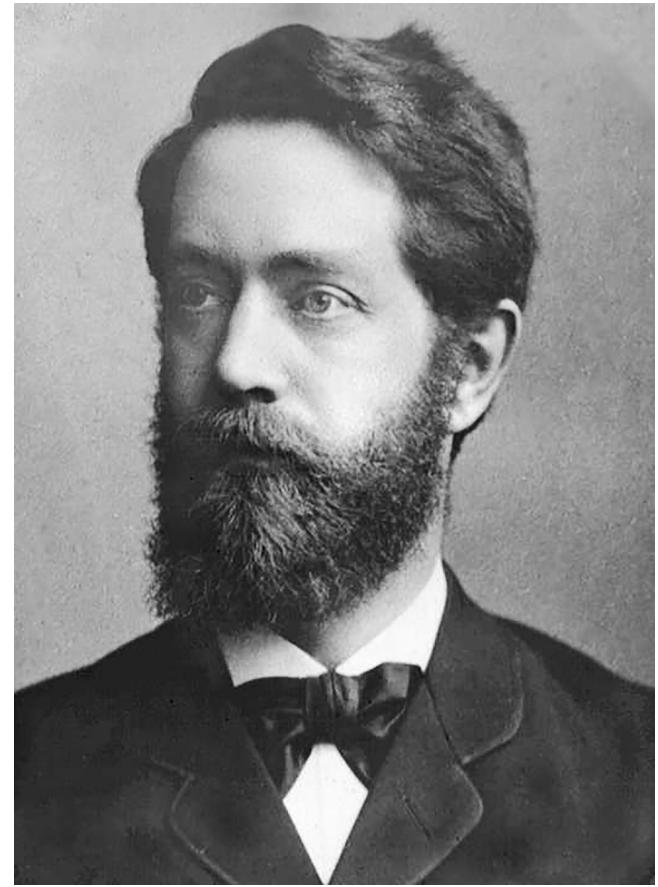

Figure 6. Felix Klein.

The first book entirely devoted to Kummer surfaces that combines geometric, algebraic, and transcendental approaches to their study was published by Ronald Hudson in 1905 [Hud05].

As I understand, Kummer's interest in 16-nodal quartic surfaces arose from his pioneering study of twodimensional families (congruences) of lines in $\mathbb{P}^{3}$. They are naturally parameterized by irreducible surfaces in the Grassmann variety $G_{1}\left(\mathbb{P}^{3}\right)$ of lines in $\mathbb{P}^{3}$. In [Kum66] Kummer gives a classification of quadratic line congruences (i.e., congruences of lines such that through a general point $x \in \mathbb{P}^{3}$ passes exactly two lines from the congruence). Let $n$ be the class of the congruence, i.e., the number of lines of the congruence that lie in a general plane. Kummer had shown that $2 \leq n \leq 7$ and when $n=2$ all the lines are tangent to a Kummer quartic surface at two points. There are six congruences like that whose lines are tangent to the same Kummer surface.

In 1870, Felix Klein in his dissertation develops a beautiful relationship between the Kummer surfaces and quadratic line complexes [Kle70]. A quadratic line complex is the intersection $X=G \cap Q$ of the Grassmann quadric $G=G_{1}\left(\mathbb{P}^{3}\right)$ in the Plücker space $\mathbb{P}^{5}$ with another quadric hypersurface. For any point $x \in \mathbb{P}^{3}$ the set of lines passing through $x$ is a plane $\sigma_{x}$ contained in $G$. Its intersection with $X$ is a conic. The locus of points $x$ such that this conic becomes reducible is the singular surface of the complex, and Klein had shown that it is a Kummer surface if the intersection $G \cap Q$ is transversal. Its 16 nodes correspond to points where $\sigma_{x} \cap X$ is a double line. In fact, Klein shows 
that the set of lines in $X$ is parameterized by the Jacobian of $C, \operatorname{Jac}(C)$, where $C$ is the Riemann surface of genus 2, the double cover of $\mathbb{P}^{1}$ realized as the pencil of quadrics spanned by $G$ and $Q$ ramified along six points corresponding to six singular quadrics in the pencil. The Kummer surface is isomorphic to $\operatorname{Kum}(\operatorname{Jac}(C))$. By a simultaneous diagonalization of quadrics $G$ and $Q$, Klein shows that the Kummer surface admits a birational nonsingular model as a complete intersection of three quadrics

$$
\sum_{i=1}^{6} x_{i}^{2}=\sum_{i=1}^{6} a_{i} x_{i}^{2}=\sum_{i=1}^{6} a_{i}^{2} x_{i}^{2}=0,
$$

where $y^{2}=\left(x-a_{1}\right) \cdots\left(x-a_{6}\right)$ is the genus 2 Riemann surface $C$ from above. Instead of 16 nodes and 16 trope-conics we now have two sets of 16 skew lines that form an abstract incidence configuration $\left(16_{6}\right)$ isomorphic to the Kummer configuration of 16 nodes and 16 trope-conics. Klein also shows that Fresnel's wave surface is characterized by the condition that $C$ is bielliptic, i.e., it admits an involution with quotient an elliptic curve. By degenerating the quadratic complex to a tetrahedral quadratic complex one obtains Cayley's tetraedroid quartic surface. In Göpel's equation of a Kummer surface from above this corresponds to vanishing of the coefficient $E$. Jessop's book [Jes03] gives an exposition of the works of Kummer and Klein on the relationship between line geometry and Kummer surfaces.

Klein's equations of a Kummer surface exhibit obvious symmetry defined by changing signs of the variables. These symmetries generate an elementary abelian 2-group $2^{5}$, the direct sum of five copies of the cyclic group of order 2 . The quartic model also admits 16 involutions $t_{i}$ defined by the projection from the nodes $p_{i}$ (take a general point $x$, join it with the node $p_{i}$, and then define $t_{i}(x)$ to be the residual intersection point). They are birational transformations, i.e., defined by an invertible rational change of variables which may be not defined on some locus of zeros of a finite set of polynomials. In 1886 Klein asked whether the group of birational automorphisms $\operatorname{Bir}(X)$ of a (general) Kummer surface is generated by the group $2^{5}$ and the projection involutions [Kle86]. In the remainder, we will discuss the progress on this problem.

In 1901 John Hutchinson, using the theory of theta functions, showed that a choice of one of 60 Göpel tetrad of nodes (means the tetrahedron with the tetrad as its vertices has no trope-conics on its faces ) leads to an equation of the Kummer surface of the form

$q\left(x_{1} x_{2}+x_{3} x_{4}, x_{1} x_{3}+x_{2} x_{4}, x_{1} x_{4}+x_{2} x_{3}\right)+c x_{1} x_{2} x_{3} x_{4}=0$,

where $q$ is a quadratic form in three variables [Hut01]. He observed that the transformation $x_{i} \mapsto 1 / x_{i}$ leaves this equation invariant, and hence defines a birational involution of the Kummer surface.

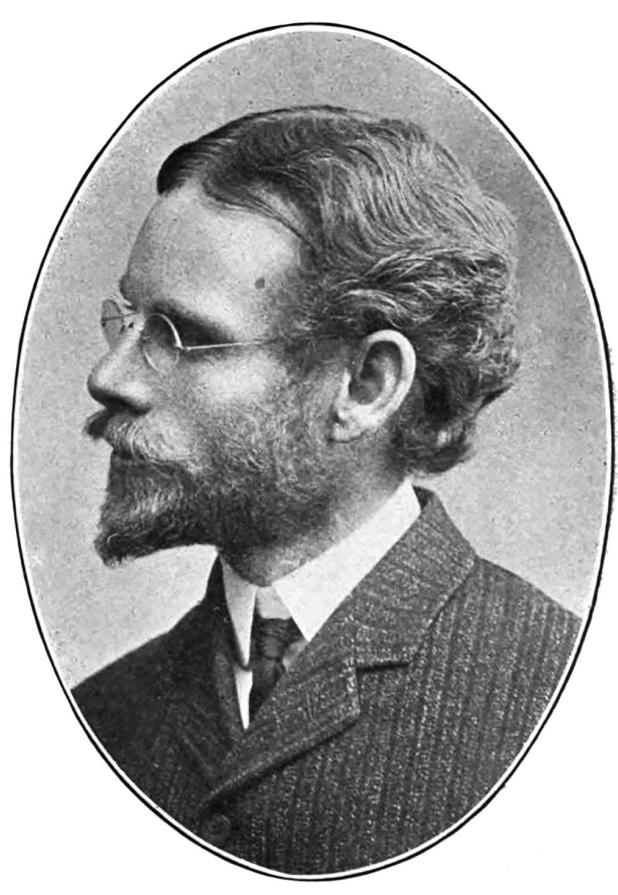

Figure 7. John Hutchinson.

He proves that these 60 transformations generate an infinite discontinuous group. However, Hutchinson did not address the question of whether adding these new transformations to Klein transformations would generate the whole group $\operatorname{Bir}(X)$.

In 1850 Thomas Weddle, correcting a mistake of Michel Chasles, noticed that the locus of singular points of quadric surfaces passing through a fixed set of six general points in $\mathbb{P}^{3}$ is a quartic surface with singular points at the six points [Wed50]. It contains 15 lines joining pairs of the points and the unique twisted cubic curve through the six nodes (it seems that Chasles asserted that there is nothing else except the twisted cubic). Different equations of the Weddle surface were given by Arthur Cayley in 1861, Carl Hierholzer in 1871, and Eugen Hunuady in 1882. The simplest equation was given later by $F$. Caspari [Cas91]:

$$
\operatorname{det}\left(\begin{array}{llll}
a a^{\prime} y z w & x & a & a^{\prime} \\
b b^{\prime} x z w & y & b & b^{\prime} \\
c c^{\prime} x y w & z & c & c^{\prime} \\
d d^{\prime} x y z & w & d & d^{\prime}
\end{array}\right)=0 .
$$

Here the six points are the points $(1: 0: 0: 0),(0$ : $1: 0: 0),(0: 0: 1: 0),(0: 0: 0: 1)$ and two points with coordinates given in the last two columns of the matrix. It is immediate to see that, after change of variables $x=a a^{\prime} x^{\prime}, y=b b^{\prime} y^{\prime}, z=c c^{\prime} z, w=d d^{\prime} w^{\prime}$, the same inversion transformation used by Hutchinson leaves the Weddle surface invariant.

In 1889 Friedrich Schottky, using the theory of theta functions, proved that a Weddle surface is birationally isomorphic to a Kummer surface [Sch89]. 


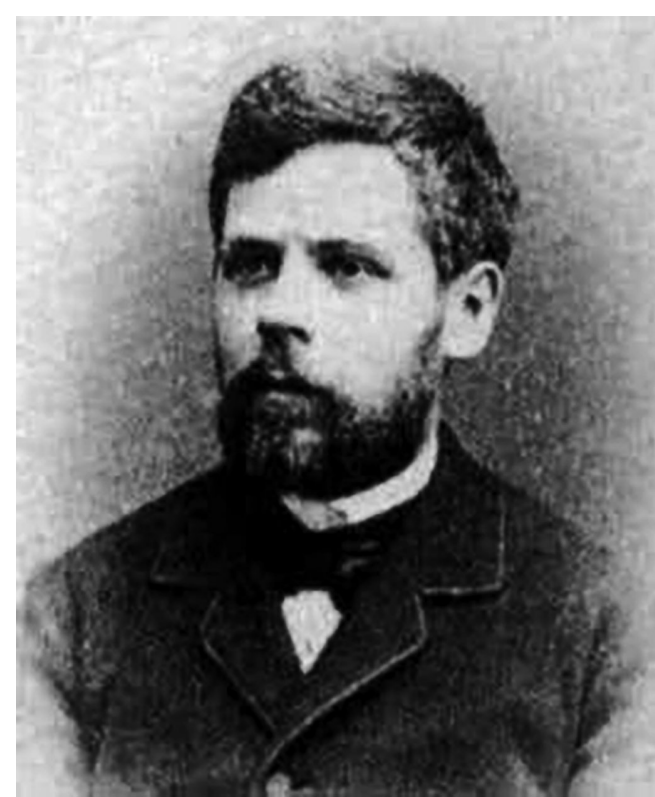

Figure 8. Friedrich Schottky.

This implies that the groups of birational automorphisms of a Weddle surface and a Kummer surface are isomorphic. For example, in 1911 Virgil Snyder gave many geometric constructions of involutions of the Weddle surface that give corresponding involutions of the Kummer surface. ${ }^{1}$ In a paper of 1914, Francis Sharpe and Clide Craig pioneered the new approach to study birational automorphisms of algebraic surfaces based on Francesco Severi's Theory of the Base. It consists of representing an automorphism of an algebraic surface as a transformation of the group of algebraic cycles on the surface. This gave an easy proof of Hutchinson's result that 60 Göpel-Hutchinson involutions generate an infinite group.

The Hutchinson involutions act freely on a nonsingular model of the Kummer surface and the quotient by these involutions are special Enriques surfaces. Enriques surfaces were discovered by Federigo Enriques in 1894 and, together with K3 surfaces, now occupy an important role in the theory of algebraic surfaces. In their paper of 2013 under the title "Enriques surfaces of Hutchinson-Göpel type and Mathieu automorphisms" Shigeru Mukai and Hasinori Ohashi study some finite groups of automorphisms of Enriques surfaces.

John Hutchinson also discovered the following amazing fact. A tetrad of nodes is called a Rosenhain tetrad if any three nodes in the tetrad lie on a trope-conic. A Weber hexad is the symmetric sum of a Göpel tetrad and a Rosenhain tetrad. Hutchinson proves that the linear system of quadric surfaces through a Weber hexad of nodes on a Kummer surface defines a birational isomorphism to the

$\overline{1_{J} \text { John Hutchinson, Virgil Snyder, Francis Sharpe, and Clide Craig were on the }}$ faculty of the Cornell Mathematics Department.
Hessian surface of a nonsingular cubic surface $F_{3}(x, y, z, w)=0$ (the Hessian surface defined by the Hessian matrix of $\left.F_{3}(x, y, z, w)\right)$ [Hut00]. The Hessian surface can also be defined as the locus of singular polar quadrics of the surface (quadrics given by linear combinations of partial derivatives of $F_{3}$ ). The rational map that assigns to a singular quadric its singular point defines a fixed-pointfree involution on a nonsingular model of the Hessian surface with quotient isomorphic to an Enriques surface. There are 192 Weber hexads, each defines a birational involution of a Kummer surface, called the Hutchinson-Weber involution. The automorphism groups as well as complex dynamics of the Hessian quartic surfaces are the subjects of study of several recent papers.

It is not surprising that none of the classical geometers could decide whether a given finite set of birational transformations generates the group $\operatorname{Bir}(X)$ of birational automorphisms of $X$. It had to wait until the end of the century for new technical tools to arrive.

A nonsingular birational model of a Kummer surface is an example of a K3 surface. By definition, a K3 surface $Y$ is a compact analytic simply connected surface with trivial first Chern class $c_{1}(Y)$. Its second Betti number is equal to 22. By a theorem of John Milnor, its homotopy type is uniquely determined by the quadratic form expressing the cup-product on $H^{2}(Y, \mathbb{Z}) \cong \mathbb{Z}^{22}$. It is a unique unimodular even quadratic lattice of signature $(3,19)$ isomorphic to the orthogonal sum of two copies of the even rank 8 negative definite unimodular lattice $E_{8}(-1)$ and three copies of the integral hyperbolic plane $U$. All K3 surfaces are diffeomorphic and realize the homotopy type defined by this lattice. Any complex K3 surface admits a Kähler metric and, by Yau's theorem, it also admits a Ricci-flat metric. It is not known how to write it explicitly (a problem of great importance for physicists). The Kummer surface is flat in this metric outside the singular points, but still one does not know how to extend this flat metric to a Ricci-flat metric on $Y$.

The quadratic lattice of cohomology $H^{2}(Y, \mathbb{Z})$ of an algebraic K3 surface contains a sublattice $S_{Y}=H^{2}(Y, \mathbb{Z})_{\text {alg }} \cong$ $\mathbb{Z}^{\circ}$ of algebraic 2 -cycles. It contains $c_{1}(L)$, where $L$ is an ample line bundle. The signature of $S_{Y}$ is equal to $(1, \rho-1)$ and, in general, it is not a unimodular lattice. The group $\operatorname{Bir}(X)$ is isomorphic to the group $\operatorname{Aut}(Y)$ of biregular automorphisms of $Y$ and it admits a natural representation $\rho: \operatorname{Aut}(Y) \rightarrow \mathrm{O}\left(H^{2}(Y, \mathbb{Z})\right)$ in the orthogonal group of $H^{2}(Y, \mathbb{Z})$ that leaves $S_{Y}$ invariant.

The fundamental Global Torelli Theorem for K3 surfaces of Ilya I. Pyatetsky-Shapiro and Igor R. Shafarevich (its original proof relies heavily on the theory of Kummer surfaces) allows one to describe the image of homomorphism $\rho$ as the set of all isometries of $H^{2}(Y, \mathbb{Z})$ that leave (after complexification) $H^{2,0}(Y, \mathbb{C})$ invariant and also leave 
invariant the semi-group of cohomology classes of holomorphic curves on $Y$. The group of automorphisms Aut $(Y)$ acts naturally on the lattice $S_{Y}$ and on the hyperbolic space $\mathbb{\square} \mathbb{M}^{\rho-1}$ associated with the linear space $S_{Y} \otimes \mathbb{R}$ of signature $(1, \rho-1)$. In this way it is realized as a discrete group of motions of a hyperbolic space. By using an isometric embedding of $S_{Y}$ into the unimodular even lattice $I I_{1,25}$ of signature $(1,25)$ isomorphic to the orthogonal sum of the Leech lattice and the hyperbolic plane $U$, Richard Borcherds introduced a method that in several cases allows one to compute the automorphism group of a K3 surface using the isometries of $I I_{1,25}$ defined by the reflections into Leech roots. In 1998, based on Borcherds' ideas, Shigeyuki Kondo proved that the group of birational automorphisms of a general Kummer surface (i.e., the Jacobian surface of a general curve of genus 2) is generated by the group $2^{5}, 16$ projection involutions, 60 Hutchinson-Göpel involutions, and 192 Hutchinson-Weber involutions. ${ }^{2}$ The group of birational automorphisms of an arbitrary Kummer surface is still unknown.

For a modern exposition of the classical theory of Kummer surfaces, we refer the reader to [Dol12]. I apologize for omitting, because of the brevity of the article, many important contributions to the study of Kummer surfaces by various mathematicians in the past and in the present. Note that a search of the title "Kummer surface" in the data base of MathSciNet gives 132 items (72 of them in this century).

\section{References}

[Bor77] C. Borchardt, Ueber die Darstellung der Kummerische fläche vierter Ordnung mit sechzehnen Knoten dürch die Göpelsche biquadratische Relation zwischen vier Thetafunktionen mit zwei Variablen, J. Reine Angew. Math. 83 (1877), 234-244.

[Cas91] F. Caspari, Sur les deux formes sous lesquelles s'expriment au moyen des fonctions théta de deux arguments, les coordonnées de la surface du quatriéme degré, décrite par les sommets des cones du second ordre qui passént par six points donnés, C. R. Hébdomadaires Acad. Sci. Paris 112 (1891), 13561359.

[Dol12] Igor V. Dolgachev, Classical algebraic geometry. a modern view, Cambridge University Press, Cambridge, 2012. MR2964027

[Fre] A. Fresnel, Oeuvres compl'etes d'Augustin Fresnel, Imprimerie Impériale, Paris. 1866, par M. M. Henri de Sanarmont, Émile Verdet, et Léonor Fresnel.

[Göp47] A. Göpel, Theoriae transcendentium abelianarum primi ordinis adumbratio levis, J. Math. Pures Appl. 35 (1847), 277-312.

\footnotetext{
${ }^{2}$ In fact, Kondo includes in the set of generators some new automorphisms of infinite order discovered by JongHae Keum instead of Hutchinson-Weber involutions. It was later observed by Ohashi that one can use Hutchinson-Weber involutions instead of Keum's automorphisms.
}

[Ham37] W. Hamilton, Third supplement to an essay on the theory of systems of rays, Trans. Royal Irish Acad. 17 (1837), $1-144$.

[Hud05] R. W. H. T. Hudson, Kummer's quartic surface, Cambridge University Press, Cambridge, 1905. Reprinted in 1990 with a forward by W. Barth. MR1097176

[Hut00] J. Hutchinson, The Hessian of the cubic surface. II, Bull. Amer. Math. Soc 6 (1900), 328-337. MR1557720

[Hut01] J. Hutchinson, On some birational transformations of the Kummer surface into itself, Bull. Amer. Math. Soc 7 (1901), 211-217. MR1557786

[Jes03] C. Jessop, A treatise of the line complex, Cambridge University Press, Cambridge, 1903. Reprinted by Chelsea Publ. Co., New York, 1969. MR0247995

[Kle70] F. Klein, Zur Theorie der Liniencomplexe des ersten und Zwieter Grades, Math. Ann. 2 (1870), 198-226.

[Kle86] F. Klein, Ueber Configurationen, welche der Kummerschen Fläche zuglecih eingeschrieben und umgeschriben sind, Math. Ann. 27 (1886), 106-142.

[Knö86] H. Knörrer, Die Fresnesche Wellenfläche, Arithmetik und Geometrie. Mathematische Miniaturen, 3, Birkhüser Verlag, Basel, 1986. MR0879281

[Kum64] E. Kummer, Ueber die Flächen vierten Grades mit sechszehn singulären Punkten, Monatsberichte Berliner Akad. 6 (1864), 246-260.

[Kum66] E. Kummer, Ueber die algebraische Strahlensysteme insbesondere die erste und zweiten Ordnung, Monatsberichte Berliner Akad. 8 (1866), 1-120.

[Lor96] G. Loria, Il passato ed il presente delle principali theorie geometriche, Carlo Clausen, Torino, 1896. MR0879281

[Sch89] F. Schottky, Ueber die Beziehungen zwischen den sechzehn Thetafuncktionen von zwei Variablen, J. Reine Angew. Math. 105 (1889), 233-244.

[Wed50] T. Weddle, On theorems in space analogous to those of Pascal and Brianchon in a plane, Cambridge and Dublin Quarterly Math. J. 5 (1850), 58-69.

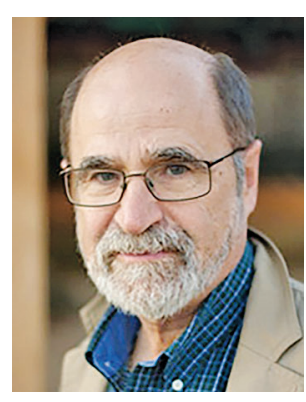

Igor Dolgachev

Credits

Opening image is courtesy of Claudio Rocchini. Licensed under the Creative Commons Attribution-Share Alike 3.0 Unported license (https://creativecommons.org /1icenses/by-sa/3.0/deed.en.

Figures 1-6 and 8 are courtesy of Wikimedia Commons. Figure 7 is courtesy of Internet Archive.

Photo of Igor Dolgachev is courtesy of Igor Dolgachev. 